\title{
Modelos para estimar biomasa de Euphorbia antisyphilitica zucc. en seis municipios de Coahuila
}

\author{
Models to estimate biomass of Euphorbia antisyphilitica Zucc. in six \\ townships of Coahuila
}

\author{
Adrián-Hernández-Ramos', Antonio Cano-Pineda', Celestino Flores-López², Jonathan Hernández-Ramos³, \\ Xavier García-Cuevas ${ }^{3}$, Martin Martínez-Salvador ${ }^{4}$ y Luis Martínez Ángel ${ }^{5}$
}

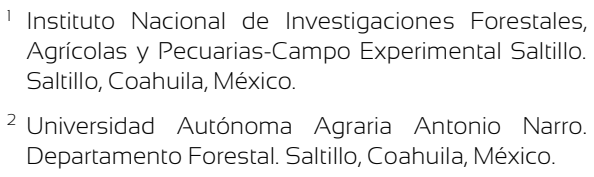

Instituto Nacional de Investigaciones Forestales, Agrícolas y Pecuarias-Campo Experimental Saltillo. Saltillo, Coahuila, México.

2 Universidad Autónoma Agraria Antonio Narro. Departamento Forestal. Saltillo, Coahuila, México

\author{
${ }^{3}$ Instituto Nacional de Investigaciones Forestales, \\ Agrícolas y Pecuarias. Campo Experimental \\ Chetumal. Chetumal, Quintana Roo, México \\ * Autor de correspondencia. cele64@gmail.com
}

\author{
${ }^{4}$ Universidad Autónoma de Chihuahua. Facultad de \\ Zootecnia y Ecología. Chihuahua, Chihuahua, \\ México. \\ ${ }^{5}$ Asesor técnico independiente.
}

\section{RESUMEN}

El escaso conocimiento sobre el crecimiento y desarrollo de las especies de zonas áridas y semiáridas en México ha generado un sobreaprovechamiento. Entre las especies más importantes económicamente en Coahuila está la candelilla, cuya información técnica disponible para la toma de decisiones en el manejo es limitada. Por lo anterior, los objetivos del presente trabajo fueron a) agrupar seis municipios con base en la producción individual de biomasa evaluada en 10 poblaciones naturales de Euphorbia antisyphilitica Zucc. y b) ajustar modelos alométricos para estimar la biomasa de E. antisyphilitica en diferentes condiciones de crecimiento, en el centro y sur de Coahuila. Con un análisis de varianza y la diferencia mínima significativa, se agruparon los municipios; para cada grupo se ajustaron modelos, mediante el método de mínimos cuadrados ordinarios. Se obtuvieron tres grupos de producción de biomasa por individuo (GI, GII y GIII). Los modelos para estimar la biomasa total verde (kilogramo por individuo) para cada grupo, resultaron con $\mathrm{R}^{2}$ Ajus superiores a 0.86, en los primeros dos grupos, y de 0.64 para GIII. La RCME (raíz del cuadrado medio del error) fue inferior a 0.425 y los parámetros de los modelos fueron significativos ( $\mathrm{p}<0.05$ ), en los cuatro casos. Además, los cuatro modelos seleccionados presentaron normalidad, homogeneidad de varianzas y la inexistencia de colinealidad entre variables. Los modelos seleccionados son estadísticamente confiables para estimar la biomasa por individuo de cada grupo considerado en este estudio, por lo que podrán ser una herramienta útil en la elaboración de planes de manejo para el aprovechamiento de candelilla.

PALABRAS CLAVE: biomasa verde, ecuaciones alométricas, estudios técnicos, manejo forestal.

\section{ABSTRACT}

The scarce knowledge about the growth and development of the species of arid and semiarid zones in Mexico, has generated an overuse. Among the most important economically in Coahuila is the candelilla, of which the technical information available for decisionmaking in management is limited. Therefore, the objectives were: a) to group six municipalities based on the individual production of biomass evaluated in 10 natural populations of Euphorbia antisyphilitica Zucc. and, b) adjust allometric models to estimate the biomass of E. antisyphilitica in different growing conditions in the center and south of Coahuila. With an analysis of variance and the minimum significant difference, the municipalities were grouped; for each group, models were adjusted, using the ordinary least squares method. Three biomass production groups were obtained per individual (GI, GII and GIII). The models to estimate the total green biomass (kilogram per individual) for each group, resulted with an $\mathrm{R}^{2}$ Ajus higher than 0.86 , in the first two groups, and for GIII it was 0.64 , the RMSE (root mean square error) was lower than 0.425 and the parameters of the models were significant $(p<0.05)$, in the four cases. In addition, the four selected models presented normality, homogeneity of variances and the nonexistence of collinearity between variables. The selected models are statistically reliable to estimate the biomass by individuals of each group considered in this study, so they can be a useful tool in the development of management plans for the use of candelilla.

KEYWORDS: green biomass, allometric equations, technical studies, forest management. 


\section{INTRODUCCIÓN}

Las regiones áridas y semiáridas de México representan aproximadamente $54.3 \%$ de la superficie del país (Cervantes, 2002) dominadas por matorral xerófilo, el cual tiene una extensión de 70.49 millones de hectáreas y representa $29.7 \%$ de la superficie nacional (Challenger y Soberón, 2008); además, son la fuente principal de productos forestales no maderables (PFNM) como son ceras, fibras, leña, entre otros. Dentro del grupo, la producción de cera de candelilla a escala nacional es de 1311 t anuales, volumen considerable en la producción de PFNM (Secretaria de Medio Ambiente y Recursos Naturales [Semarnat], 2016). Este producto es extraído de la candelilla (Euphorbia antisyphilitica Zucc.), especie con una amplia distribución y que presenta rendimientos en producción variados, de acuerdo con las condiciones ambientales en las que se desarrolla (Organización de las Naciones Unidas para la Agricultura y la Alimentación [FAO], 2004; Limones-Rubio, Aguirre-Ureña, Fonseca-Espino, Muro y Sánchez, 2015).

A pesar de la importancia de estos ecosistemas y de las especies comerciales que en ellos se desarrollan, aún no se cuenta con el conocimiento sobre su dinámica de crecimiento y desarrollo. Esto ha generado un aprovechamiento irracional de los recursos, que a largo plazo causa entre otros tipos de degradación, el deterioro de las comunidades vegetales, así como la reducción de la superficie de distribución (FAO, 2004). En este contexto, es necesario generar técnicas estadísticas confiables que ayuden a determinar la dinámica de crecimiento, su rendimiento y las relaciones intraespecíficas de especies no maderables bajo aprovechamiento. Una de las especies económicamente más importantes en el estado de Coahuila es la candelilla, cuya información técnica disponible para la toma de decisiones en el manejo forestal es limitada (Zamora, Velasco, Cano y Arellano, 2008; Semarnat, 2013).

Entre dichas limitantes técnicas, se encuentra la insuficiencia de información relacionada con la producción de biomasa (Aristizábal, 2011), en especial para especies forestales desarrolladas en ecosistemas áridos y semiáridos
(Méndez, Turlan, Ríos y Nájera, 2012); ya que gran parte de los estudios realizados para describir la dinámica de esta variable se agrupan en especies forestales arbóreas con importancia maderable (Soriano-Luna, Ángeles-Pérez, Martínez-Trinidad, Plascencia-Escalante y Razo-Zárate, 2015; Winck et al., 2015; Yepes et al., 2016; HernándezRamos et al., 2017; Flores et al., 2018). La biomasa en ecosistemas forestales es fijada y acumulada mediante el proceso de fotosíntesis, contribuye a las reservas de carbono en la tierra y es empleada como indicador para evaluar la productividad del sitio forestal, además, juega un papel importante en el manejo forestal sostenible de los recursos (Návar-Cháidez, 2010; Ferrere, Lupi y Boca, 2014).

Para evaluar la biomasa se utilizan métodos directos e indirectos (Aristizábal, 2011); en estos últimos, los modelos matemáticos generados a partir de relaciones alométricas son herramientas importantes de aplicación directa para estimar de forma confiable la distribución de biomasa a escala de especies y a escala de ecosistema forestal, abarcando áreas extensas (Solano, Vega, Eras y Cueva, 2014; Flores et al., 2018). Además, son aplicables para estimar la fijación de $\mathrm{CO}_{2}$ y las cargas de combustible (Ferrere et al., 2014). Estos modelos consisten en estimar una variable de difícil medición (biomasa) mediante mediciones que se pueden realizar directamente en inventarios forestales y que representan un bajo costo (Solano et al., 2014).

Las ecuaciones alométricas deben de ser desarrolladas a escala local y por especie para obtener una mejor estimación de la biomasa, ya que se presentan limitaciones al ajustarlas de manera global debido a las distintas condiciones de crecimiento en las que se desarrollan las especies forestales (Solano et al., 2014). Además, las ecuaciones específicas por sitio son de mayor utilidad dentro del manejo forestal (Návar-Cháidez, 2010). Para este tipo de estudios, lo recomendable es contar con ecuaciones alométricas por especie o por condición de crecimiento (sitio) (Carrillo, Acosta, Flores, Juárez y Bonilla, 2014). 


\section{OBJETIVOS}

Los objetivos de este trabajo fueron a) agrupar seis municipios de acuerdo con la producción individual de biomasa evaluada en 10 poblaciones naturales de $E$. antisyphilitica y b) ajustar modelos alométricos para estimar la biomasa de E. antisyphilitica en diferentes condiciones de crecimiento en el centro y sur de Coahuila.

\section{MATERIALES Y MÉTODOS}

\section{Área de estudio}

La investigación se desarrolló en los municipios de Cuatro Ciénegas, Parras de la Fuente, Ramos Arizpe, San Pedro de las Colonias, Sierra Mojada y Viesca, correspondientes a la parte centro y sur del Estado de Coahuila (Fig. 1); abarcando 10 poblaciones naturales localizadas en predios con programa de manejo forestal vigente de candelilla. Estas localidades se caracterizan por presentar climas del tipo árido y semiárido $(B s)$ y muy árido $(B w)$, con temperaturas medias anuales de $18{ }^{\circ} \mathrm{C}$ a $22{ }^{\circ} \mathrm{C}$ y precipitación anual de $100 \mathrm{~mm}$ a $400 \mathrm{~mm}$ (Instituto Nacional de Estadística y Geografía [Inegi], 2007). La altitud está comprendida entre $950 \mathrm{~m} \mathrm{y} 1500 \mathrm{~m}$. La vegetación correspondiente al área es matorral desértico micrófilo y rosetófilo (Inegi, 2016).

\section{Toma de datos en campo}

Los datos se colectaron mediante un muestreo selectivo de forma destructiva, donde a cada individuo se le midió altura media $(\mathrm{A}, \mathrm{cm})$, diámetro menor y mayor de la base $(\mathrm{cm})$ y diámetro menor y mayor de la cobertura de copa $(\mathrm{cm})$. Posteriormente la planta fue cosechada para obtener la biomasa total verde (Btv) (en kilogramos por individuo "kg individuo-1"), con una báscula Torrey de $0.005 \mathrm{~kg}$ de resolución (Winck et al., 2015; Hernández-Ramos et al., 2017; Flores et al., 2018; Villavicencio-Gutiérrez, Hernández-Ramos, Aguilar-González y García-Cuevas, 2018). La muestra se conformó de 1083 individuos distribuidos de forma que abarcaran la mayor variabilidad de condiciones de crecimiento de la especie y las categorías diamétricas existentes en las diez poblaciones muestreadas (Tabla 1).

\section{Análisis estadístico}

Agrupación de datos. La agrupación de los municipios con atributos similares se realizó mediante un análisis de varianza (Andeva), donde el factor primario fue el municipio y la variable de respuesta la Btv. Posterior al análisis de significancia entre los seis municipios, se realizó el contraste de medias mediante la técnica de la diferencia minima significativa o método LSD (least significant difference), que consiste en la prueba de hipótesis por parejas de la forma $\mathrm{H}_{0}: \mu_{\mathrm{i}}=\mu_{\mathrm{j}} ; \mathrm{H}_{1}: \mu_{\mathrm{i}} \neq \mu_{\mathrm{j}}$. Por lo tanto, las medias fueron estadísticamente diferentes si $\left(\overline{\mathrm{y}}_{i}-\overline{\mathrm{y}}_{j}\right)>L S D$, donde la mínima diferencia significativa $(L S D)$ está dada por: $L S D=$ $\tau_{\alpha / 2 ;} N-1 \sqrt{\hat{s}_{R}{ }^{2}\left(\frac{1}{n_{i}}+\frac{1}{n_{j}}\right)}$, siendo $n_{i}$ y $n_{j}$ el número de observaciones correspondientes a cada medida, $N-1$ el número de grados de libertad y $t_{a / 2 ; \mathrm{N}-1}$ el valor crítico de la distribución $t$ con $N-1$ grados de libertad, que deja una probabilidad a su derecha igual a $\alpha / 2$ (Williams y Abdi, 2010). Finalmente, se realizó la agrupación de medias por grupo de municipios (Statistical Analysis Systems Institute Inc., [SAS], 2017).

TABLA 1. Distribución de la muestra de las poblaciones de Euphorbia antisyphilitica Zucc., bajo estudio en Coahuila, México.

\begin{tabular}{lccc}
\hline No. & Municipio & Población & $\begin{array}{c}\text { Plantas } \\
(n)\end{array}$ \\
\hline \multirow{2}{*}{$\mathbf{1}$} & Cuatro Ciénegas & Estanque de León & 91 \\
& & Las Morenas & 103 \\
& Tanque Nuevo & 101 \\
\hline $\mathbf{2}$ & Parras de la & El Amparo & 170 \\
\hline \multirow{2}{*}{$\mathbf{3}$} & Fuente & Las Coloradas & 100 \\
& Ramos Arizpe & Plan de Guadalupe & 105 \\
\hline $\mathbf{4}$ & San Pedro de las & Cosme & 113 \\
\hline $\mathbf{5}$ & Colonias & Mala Noche & 120 \\
\hline $\mathbf{6}$ & Sierra Mojada & San José de Madero & 120 \\
\hline & Viesca & La Fe & 60 \\
\hline & & Total: & 1083 \\
\hline
\end{tabular}




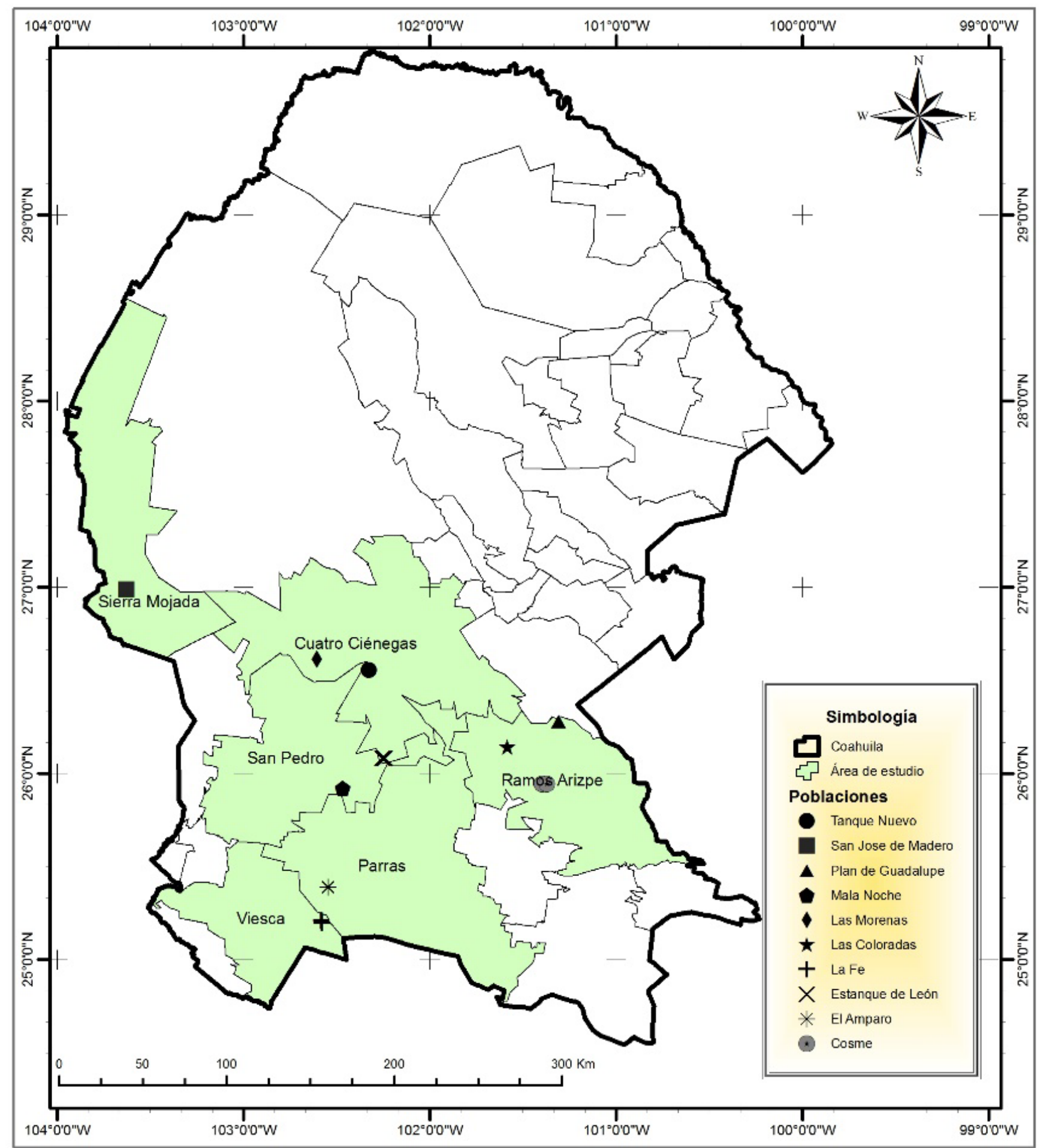

FIGURA 1. Distribución geográfica de las poblaciones de Euphorbia antisyphilitica Zucc., bajo estudio en Coahuila, México. 


\section{Selección de variables para el ajuste de modelos. El} diámetro promedio de la cobertura de copa (DPC, $\mathrm{cm}$ ) y el diámetro promedio de la base $(\mathrm{DPB}, \mathrm{cm})$ resultaron de la media de las medidas de la planta tomadas en campo para cada variable; la cobertura arbustiva $\left(\mathrm{CA}, \mathrm{m}^{2}\right)$ fue calculada con la expresión $C A=\frac{\pi}{40000} *\left(d_{1}+d_{2} / 2\right)^{2}$, donde $\mathrm{d}_{1} \mathrm{y}$ $\mathrm{d}_{2}$ son los diámetros de cobertura medidos a la planta en centímetros. Las variables antes generadas y la A fueron relacionadas con la Btv mediante un análisis de correlación de Pearson, seleccionando para el ajuste de los modelos únicamente aquellas que presentaron los valores de correlación ( $r$ ) más altos y las que aportaron mayor variabilidad en el modelo lineal ajustado mediante la técnica Stepwise (Volke, 2008). Además, se graficaron las variables independientes contra la Btv para observar su tendencia.

Depuración de bases de datos. Para la detección de los valores atípicos de la muestra, previo al ajuste de los modelos, se empleó el método de eliminación directa mediante el análisis gráfico de la dispersión de los datos y con los valores de los residuales studentizados; estos últimos se calcularon en SAS 9.4 ${ }^{\circledR}$ con PROC GLM (Modelos lineales generalizado por sus siglas en ingles) y la opción $r$ Influence (SAS, 2017). Se consideraron valores atípicos los residuales mayores al valor critico de \pm 2 , con el supuesto de que al menos $5 \%$ de la muestra no pertenecen a la población (Coenders y Saez, 2000) y se eliminaron estos valores de la base de datos para evitar tener resultados sesgados en los análisis de regresión (Chen, 1978).

Ajuste estadístico de los modelos. Se emplearon tres modelos seleccionados de la literatura, que presentaron resultados sobresalientes al estimar la biomasa de diversas especies arbóreas y arbustivas (Gómez-García, CrecenteCampo y Diéguez-Aranda, 2013; Soriano-Luna et al., 2015; Hernández-Ramos et al., 2017; Villavicencio-Gutiérrez et al., 2018) (Tabla 2). Una vez seleccionadas las variables más relacionadas con la Btv, se realizó el ajuste de los modelos, mediante el método de mínimos cuadrados ordinarios con PROC MODEL en SAS 9.3 ® (SAS, 2017) para cada uno de los grupos de municipios resultantes de la agrupación.
La selección de los modelos de mejor ajuste al conjunto de datos de Btv se efectuó de acuerdo con los mayores valores del coeficiente de determinación ajustado por el número de parámetros $\left(\mathrm{R}^{2} \mathrm{Ajus}\right)$, el cual mide la variabilidad explicada por el modelo, y los menores de la raíz del cuadrado medio del error (RCME), que proporciona la media de las diferencias entre los valores estimados y los observados (Tlaxcala-Méndez, SantosPosadas, Hernández-de la Rosa y López-Ayala, 2016), además de la significancia de sus parámetros y del coeficiente de determinación entre los valores predichos y observados (Tlaxcala-Méndez et al., 2016; Flores et al., 2018). Los supuestos de regresión del modelo se analizaron con la prueba de Shapiro-Wilk para la normalidad de los datos, la prueba de White para detectar la homocedastisidad de los residuales y la prueba de Durbin-Watson para detectar autocorrelación de los errores. Por último, a los modelos se les evaluó la capacidad predictiva mediante el sesgo promedio absoluto $(\bar{E})$ en kilogramos de Btv y la diferencia agregada en porcentaje $(D A \%)$ (Trincado y Leal, 2006; Tlaxcala-Méndez et al., 2016; Hernández-Ramos et al., 2017). El análisis estadístico se realizó en el programa SAS 9.3 尺.

$$
\operatorname{Sesgo}(\bar{E})=\frac{\sum_{i=1}^{n}\left(Y_{i}-\hat{Y}_{i}\right)}{n} ; \quad D A \%=\left(\frac{\bar{E}}{\bar{Y}_{i}}\right) * 100
$$

Donde: $Y_{i}$ es el valor observado, $\hat{Y}_{i}$ es el valor predicho con el modelo, $\bar{Y}_{i}$ es la media de la variable dependiente y $n$ es el número de observaciones.

TABLA 2. Modelos seleccionados para el ajuste de la biomasa verde de Euphorbia antisyphilitica Zucc., en seis municipios de Coahuila.

\begin{tabular}{ccc}
\hline Identificador & Nombre & Estructura \\
\hline 1 & Schumacher-Hall & Btv $=\beta_{0}\left(x_{i}\right)^{\beta_{1}}\left(x_{j}\right)^{\beta_{2}}$ \\
2 & $\begin{array}{c}\text { Schumacher } \\
\text { exponencial }\end{array}$ & $B t v=\beta_{0} \operatorname{Exp}^{\left(-\beta_{1} / x_{i}\right)}$ \\
3 & Spurr & $B t v=\beta_{1}\left(x_{i}{ }^{2} x_{j}\right)^{\beta_{2}}$ \\
\hline
\end{tabular}

Btv: Biomasa total verde (kg individuo $\left.{ }^{-1}\right) ; x_{i}, x_{i}$ : variables independientes; $\beta_{i}$ : coeficientes de regresión. 


\section{RESULTADOS}

\section{Agrupación de datos de biomasa}

El análisis de varianza indica diferencias significativas entre los municipios y entre las poblaciones, el error Tipo III en ambos es significativo $(\alpha=0.0001)$. Mientras que los resultados obtenidos con LSD muestran una agrupación de los municipios en tres grupos de acuerdo con la Btv por individuo. El municipio de Parras de la Fuente (El Amparo) formó el primer grupo (GI) con la mayor media registrada igual a $1.722 \mathrm{~kg}$ individuo ${ }^{-1}$, el segundo grupo (GII) lo conformaron las poblaciones de los municipios de San Pedro (Mala Noche), Cuatro Ciénegas (Estanque de León, Las Morenas y Tanque Nuevo), Ramos Arizpe (Las Coloradas, Plan de Guadalupe y Cosme) y Viesca (La Fe), con medias similares (1.046, 0.924, 0.917 y 0.809$) \mathrm{kg}$ individuo-1, respectivamente; por último, el grupo tres (GIII) se conformó por la población colectada en el municipio de Sierra Mojada con $0.657 \mathrm{~kg}$ individuo- ${ }^{-1}$, siendo este valor de Btv el más bajo de las poblaciones evaluadas (Fig. 2).

El municipio de Parras de la Fuente presentó valores altos de biomasa (GI) y Sierra Mojada con la población de San José de Madero presentó los valores más bajos (GIII), comparados con el resto del listado, por ello, se clasificó en un grupo específico a cada una de estas poblaciones con el fin de disminuir la variabilidad de estimación al momento de ajustar los modelos de regresión. En cuanto al GII, se integró con cuatro municipios con condiciones de biomasa similares. Las poblaciones que presentaron los mayores valores de biomasa fueron El Amparo y Las Coloradas con $1.722 \mathrm{~kg}$ individuo-1 y $1.507 \mathrm{~kg}^{-1}$ individuo ${ }^{-1}$, respectivamente. Las poblaciones menos productivas fueron San José de Madero, Estanque de León y Plan de Guadalupe con $0.657 \mathrm{~kg}$ individuo ${ }^{-1}, 0.526 \mathrm{~kg}$ individuo ${ }^{-1} \mathrm{y}$ $0.420 \mathrm{~kg}$ individuo $^{-1}$, para cada una; el resto de las poblaciones oscilaron entre $1.178 \mathrm{~kg}$ individuo ${ }^{-1}$ a $0.809 \mathrm{~kg}$ individuo ${ }^{-1}$.

\section{Selección de variables relacionadas con la biomasa verde}

Las variables correlacionadas con la Btv son la CA (0.90), la A por DPC (0.88), el DPC (0.87) y el DPB (0.78), considerando una relación de tipo lineal. Sin embargo, la dispersión de los datos es de tipo sigmoidal por lo que se analizaron con una regresión Stepwise, indicando que las variables A, A por DPB (ADPB), DPC, ADPC y cobertura arbustiva son significativas (0.05) dentro del modelo. Las dos pruebas coincidieron en que las variables A, DPC, DPB y la CA son adecuadas para estimar la Btv (Fig. 3), con ellas se ajustaron los modelos seleccionados, además se integraron las iteraciones entre las variables.

La representación gráfica de los valores observados de Btv contra las variables independientes A, DPC y DPB indican una tendencia exponencial que es representada con el tipo de modelos no lineales propuestos, caso contrario para la cobertura, que tiene una relación lineal con la Btv, razón por la que no se consideró en el ajuste de los modelos, además de que es una variable calculada con el DPC, con el cual esta correlacionado.

\section{Modelos de biomasa verde}

El ajuste de los modelos se realizó para cada grupo (GI, GII y GIII). Los modelos seleccionados presentaron una $\mathrm{R}^{2}$ Ajus superior a 0.86 , en los primeros dos grupos y, para GIII, fue de 0.64. La RCME fue inferior a 0.425 y los parámetros de los modelos fueron todos significativos $(p<0.05)$. Los modelos seleccionados para estimar la Btv de cada grupo quedaron de la siguiente forma: para GI el modelo 2 con las variables DPB y DPC (Tabla 3); para GII los modelos con mejor ajuste y factibles para estimar la Btv de candelilla fueron 1 y 2 con las variables DPC, DPB y A (Tabla 4); finalmente, para el GIII fue el modelo 7, que emplea el DPC como variable independiente; aunque en este grupo los modelos restantes, a excepción del 6 , presentaron valores de los estadísticos superiores al modelo 7, estos se descartaron por no mostrar significancia en uno de sus parámetros (Tabla 5). 

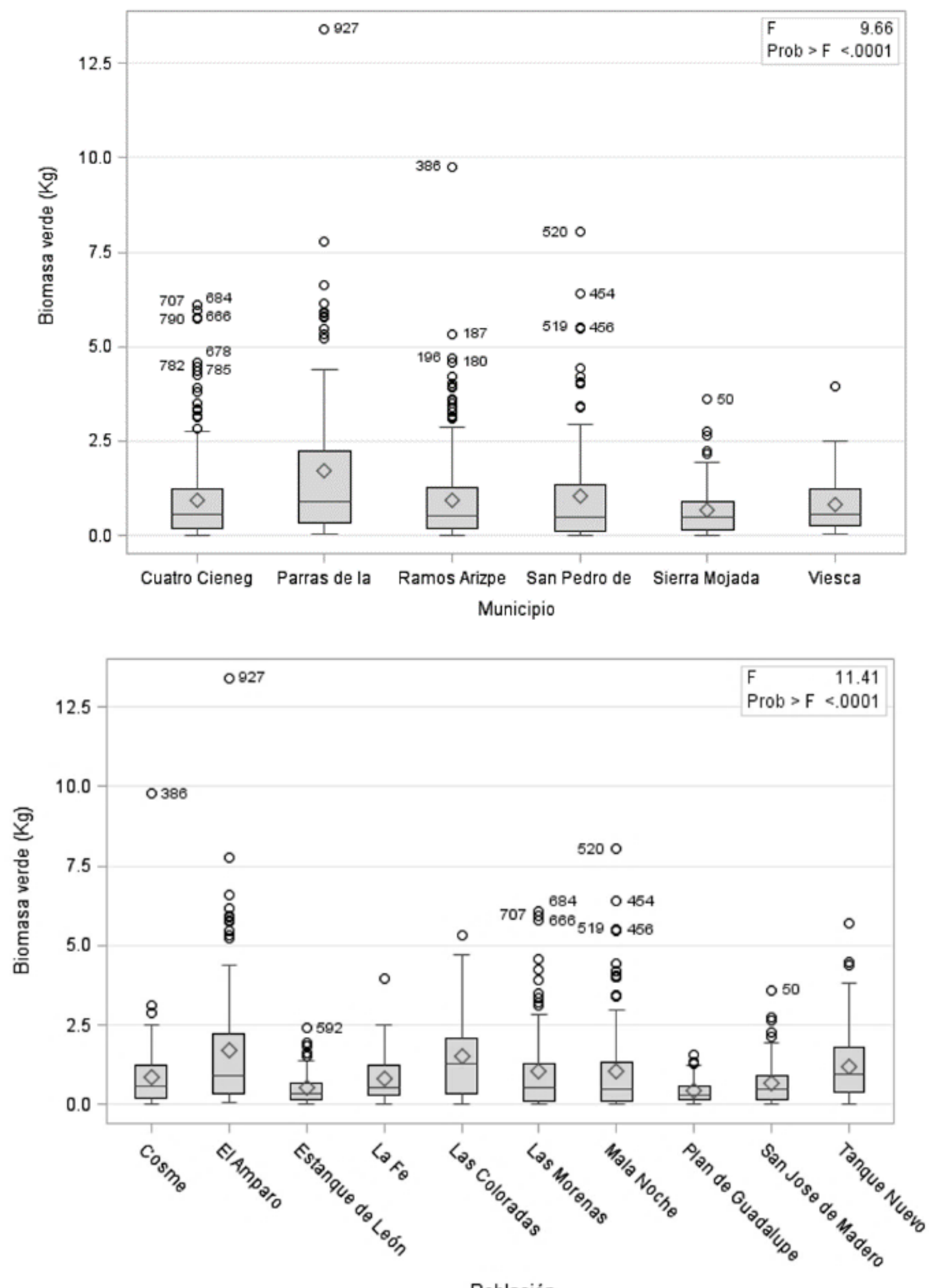

Población

FIGURA 2. Biomasa verde promedio por municipio (arriba) y por población (abajo) de Euphorbia antisyphilitica Zucc. 


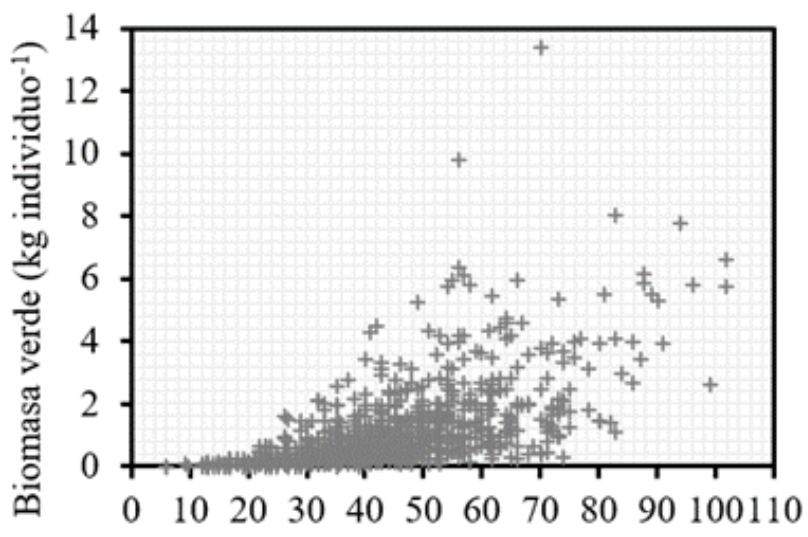

Altura $(\mathrm{cm})$

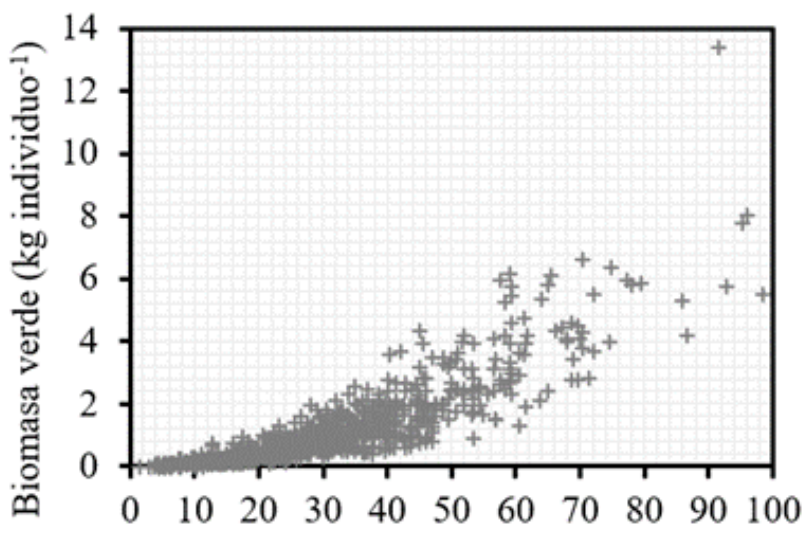

Diámetro promedio de la cobertura $(\mathrm{cm})$
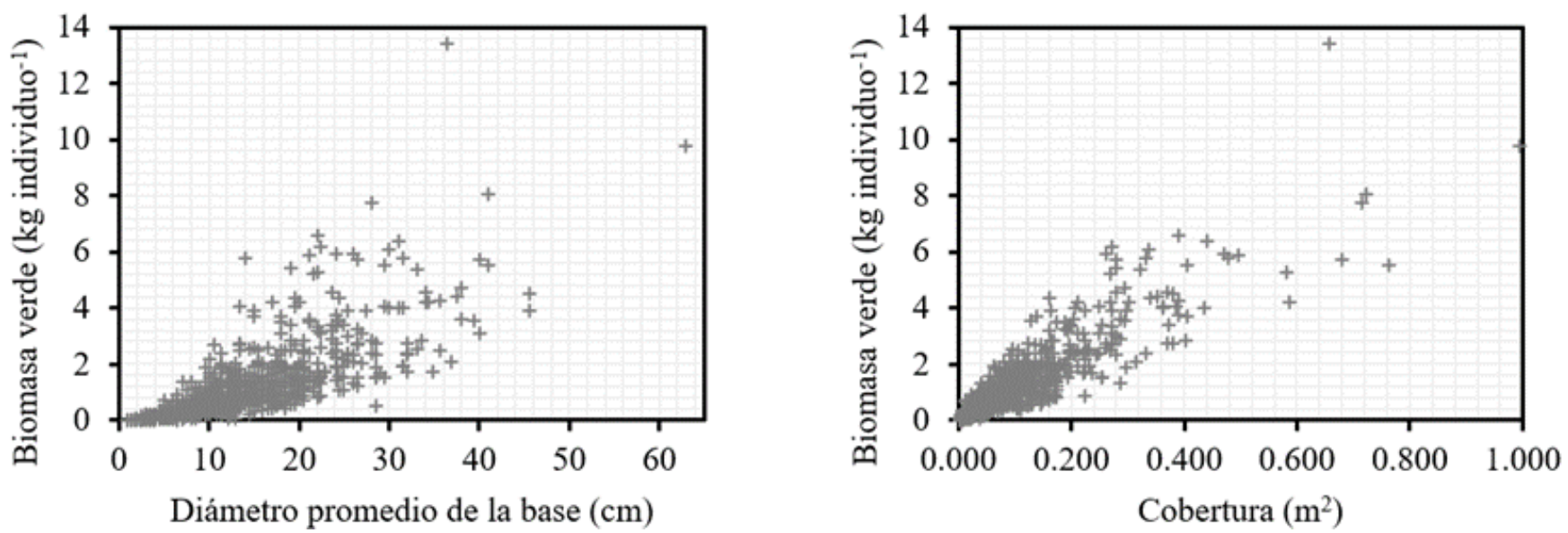

FIGURA 3. Relación entre la biomasa verde (kg) y las variables explicativas con mayor influencia en el modelo.

TABLA 3. Estadísticos de ajuste de los modelos analizados para los datos del conjunto GI, para estimar la biomasa verde de Euphorbia antisyphilitica Zucc.

\begin{tabular}{|c|c|c|c|c|c|c|c|}
\hline No. & Modelo & RCME & $R^{2}$ ajus & Parámetro & Estimación & $\operatorname{Pr}>|t|$ & V.I. \\
\hline \multirow[t]{3}{*}{1} & Schumacher-Hall & 0.673 & 0.897 & $\beta_{0}$ & 0.003773 & 0.0005 & \multirow{3}{*}{ DPB; $A$} \\
\hline & & & & $\beta_{1}$ & 1.702584 & $<0.0001$ & \\
\hline & & & & $\beta_{2}$ & 0.453807 & $<0.0001$ & \\
\hline \multirow[t]{3}{*}{2} & Schumacher-Hall & 0.590 & 0.921 & $\beta_{0}$ & 0.004682 & $<0.0001$ & \multirow{3}{*}{$\begin{array}{l}\text { DPB; } \\
\text { DPC. }\end{array}$} \\
\hline & & & & $\beta_{1}$ & 1.127425 & $<0.0001$ & \\
\hline & & & & $\beta_{2}$ & 0.841933 & $<0.0001$ & \\
\hline \multirow[t]{2}{*}{3} & Spurr & 0.705 & 0.887 & $\beta_{0}$ & 0.00167 & $<0.0001$ & \multirow{2}{*}{ DPB; $A$} \\
\hline & & & & $\beta_{1}$ & 0.766108 & $<0.0001$ & \\
\hline \multirow[t]{2}{*}{4} & Spurr & 0.912 & 0.811 & $\beta_{0}$ & 0.000761 & 0.042 & \multirow{2}{*}{ DPC; $A$} \\
\hline & & & & $\beta_{1}$ & 0.689543 & $<0.0001$ & \\
\hline \multirow[t]{2}{*}{5} & Spurr & 0.596 & 0.919 & $\beta_{0}$ & 0.005945 & $<0.0001$ & \multirow{2}{*}{$\begin{array}{l}\text { DPB; } \\
\text { DPC. }\end{array}$} \\
\hline & & & & $\beta_{1}$ & 0.652691 & $<0.0001$ & \\
\hline
\end{tabular}

RCME: raíz del cuadrado medio del error; $R^{2}$ ajus: coeficiente de determinación ajustado; Pr>|t|: valor de significancia en la prueba de $t$; $V$.I.: variable independiente. 
TABLA 4. Estadísticos de ajuste de los modelos analizados para los datos del conjunto GII, para estimar la biomasa verde de Euphorbia antisyphilitica Zucc.

\begin{tabular}{|c|c|c|c|c|c|c|c|}
\hline No. & Modelo & RCME & $R^{2}$ ajus & Parámetro & Estimación & $\operatorname{Pr}>|t|$ & V.I. \\
\hline \multirow[t]{3}{*}{1} & \multirow[t]{3}{*}{ Schumacher-Hall } & \multirow[t]{3}{*}{0.425} & \multirow[t]{3}{*}{0.8617} & $\beta_{0}$ & 0.0011 & $<0.0001$ & \multirow{3}{*}{ DPC; $A}$. \\
\hline & & & & $\beta_{1}$ & 1.647354 & $<0.0001$ & \\
\hline & & & & $\beta_{2}$ & 0.300468 & $<0.0001$ & \\
\hline \multirow[t]{3}{*}{2} & \multirow[t]{3}{*}{ Schumacher-Hall } & \multirow[t]{3}{*}{0.3961} & \multirow[t]{3}{*}{0.8798} & $\beta_{0}$ & 0.003614 & $<0.0001$ & \multirow{3}{*}{ DPB; DPC } \\
\hline & & & & $\beta_{1}$ & 0.48315 & $<0.0001$ & \\
\hline & & & & $\beta_{2}$ & 1.268382 & $<0.0001$ & \\
\hline \multirow[t]{2}{*}{3} & \multirow[t]{2}{*}{ Spurr } & \multirow[t]{2}{*}{0.503} & \multirow[t]{2}{*}{0.8062} & $\beta_{0}$ & 0.002118 & $<0.0001$ & \multirow{2}{*}{ PDB; A. } \\
\hline & & & & $\beta_{1}$ & 0.67586 & $<0.0001$ & \\
\hline \multirow[t]{2}{*}{4} & \multirow[t]{2}{*}{ Spurr } & \multirow[t]{2}{*}{0.4477} & \multirow[t]{2}{*}{0.8465} & $\beta_{0}$ & 0.000427 & $<0.0001$ & \multirow{2}{*}{ DPC; $A$. } \\
\hline & & & & $\beta_{1}$ & 0.725157 & $<0.0001$ & \\
\hline \multirow[t]{2}{*}{5} & \multirow[t]{2}{*}{ Spurr } & \multirow[t]{2}{*}{0.4647} & \multirow[t]{2}{*}{0.8346} & $\beta_{0}$ & 0.008563 & $<0.0001$ & \multirow{2}{*}{ DPB; DPC. } \\
\hline & & & & $\beta_{1}$ & 0.54779 & $<0.0001$ & \\
\hline \multirow[t]{2}{*}{6} & \multirow[t]{2}{*}{ Schumacher-Exp } & \multirow[t]{2}{*}{0.4694} & \multirow[t]{2}{*}{0.8312} & $\beta_{0}$ & 14.53953 & $<0.0001$ & \multirow{2}{*}{ DPC. } \\
\hline & & & & $\beta_{1}$ & 86.54569 & $<0.0001$ & \\
\hline
\end{tabular}

TABLA 5. Estadísticos de ajuste de los modelos analizados para los datos del conjunto GIII, para estimar la biomasa verde de Euphorbia antisyphilitica Zucc.

\begin{tabular}{|c|c|c|c|c|c|c|c|c|}
\hline No. & Modelo & RCME & $R^{2}$ ajus & Sesgo & Parámetro & Estimación & $\operatorname{Pr}>|t|$ & V.I. \\
\hline \multirow[t]{3}{*}{1} & Schumacher-Hall & 0.3489 & 0.7151 & 0.00476 & $\beta_{0}$ & 0.000153 & 0.1666 & \multirow{3}{*}{$\begin{array}{l}\mathrm{DPC} ; \\
\mathrm{A} .\end{array}$} \\
\hline & & & & & $\beta_{1}$ & 1.611161 & $<0.0001$ & \\
\hline & & & & & $\beta_{2}$ & 0.774283 & $<0.0001$ & \\
\hline \multirow[t]{3}{*}{2} & Schumacher-Hall & 0.3694 & 0.6807 & -0.00807 & $\beta_{0}$ & 0.004172 & 0.158 & \multirow{3}{*}{$\begin{array}{l}\text { DPB; } \\
\text { DPC. }\end{array}$} \\
\hline & & & & & $\beta_{1}$ & 0.56963 & 0.0012 & \\
\hline & & & & & $\beta_{2}$ & 1.06013 & $<0.0001$ & \\
\hline \multirow[t]{2}{*}{3} & Spurr & 0.3528 & 0.7087 & -0.01207 & $\beta_{0}$ & 0.003176 & 0.0873 & \multirow{2}{*}{$\begin{array}{l}\text { DPB; } \\
\text { A. }\end{array}$} \\
\hline & & & & & $\beta_{1}$ & 0.615965 & $<0.0001$ & \\
\hline \multirow[t]{2}{*}{4} & Spurr & 0.3473 & 0.7177 & 0.00614 & $\beta_{0}$ & 0.000144 & 0.1609 & \multirow{2}{*}{$\begin{array}{l}\text { DPC; } \\
\text { A. }\end{array}$} \\
\hline & & & & & $\beta_{1}$ & 0.80028 & $<0.0001$ & \\
\hline \multirow[t]{2}{*}{5} & Spurr & 0.3779 & 0.6658 & -0.0117 & $\beta_{0}$ & 0.010447 & 0.0794 & \multirow{2}{*}{$\begin{array}{l}\text { DPB } \\
\text { DPC }\end{array}$} \\
\hline & & & & & $\beta_{1}$ & 0.490747 & $<0.0001$ & \\
\hline \multirow[t]{2}{*}{6} & Spurr & 0.4014 & 0.623 & -0.01572 & $\beta_{0}$ & 0.026451 & 0.0165 & \multirow{2}{*}{ DPB. } \\
\hline & & & & & $\beta_{1}$ & 0.641409 & $<0.0001$ & \\
\hline \multirow[t]{2}{*}{7} & Schumacher-Exp & 0.3878 & 0.648 & 0.01684 & $\beta_{0}$ & 5.125135 & $<0.0001$ & \multirow[t]{2}{*}{ DPC } \\
\hline & & & & & $\beta_{1}$ & 60.80586 & $<0.0001$ & \\
\hline
\end{tabular}


En los cuatro modelos seleccionados se verificaron los supuestos de regresión, con la prueba de Shapiro-Wilk se confirmó que los datos son tendentes a la distribución normal, ya que el valor del estadístico fue $W=0.87(\mathrm{Pr}<$ $W=0.0001$, para los modelos seleccionados), y la prueba de White comprobó la homogeneidad de varianzas. La inexistencia de autocorrelación de los errores se observó con la prueba de Durbin-Watson, al obtener valores de 2.17, 1.64 y 1.81 para GI, GII y GIII, respectivamente. Además, el análisis gráfico de los valores predichos con los cuatro modelos y de los observados muestran una tendencia recta, con una correlación lineal entre estos de
0.923 para GI, 0.862 y 0.881 de los modelos seleccionados 1 y 2 de GII y 0.653 para GIII, esto confirma un ajuste adecuado de los modelos seleccionados para estimar la Btv de candelilla en la región muestreada (Fig. 4).

La capacidad predictiva de los modelos seleccionados mostró un E y DA\% de $0.0197 \mathrm{~kg}$ (1.144\%) para GI, 0.0134 $\mathrm{kg}(1.437 \%)$ y $0.018 \mathrm{~kg}(1.927 \%)$ en GII y para GIII fue de $-0.01684 \mathrm{~kg}(-2.566 \%)$ individuo ${ }^{-1}$. En los primeros dos grupos los modelos presentan una sobreestimación de la Btv no mayor a $2 \%$ y para el grupo restante fue ligeramente mayor por la variabilidad que existe en las poblaciones evaluadas.

(a)

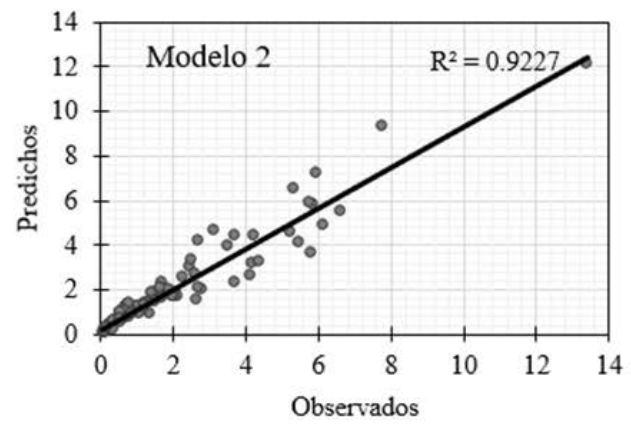

(b)
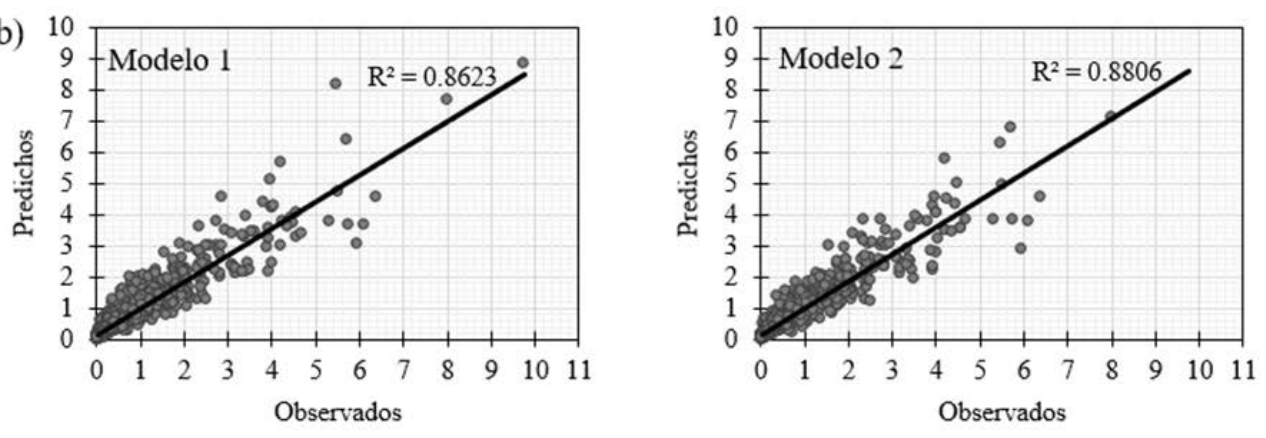

(c)

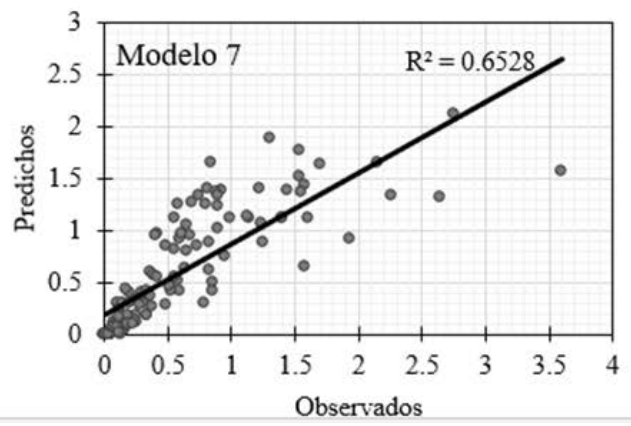

FIGURA 4. Valores de biomasa verde (Btv) estimada contra la observada para las regiones GI (a), GII (b) y GIII (c), empleando los modelos seleccionados. 


\section{DISCUSIÓN}

Las variaciones en la Btv ( $\mathrm{kg}$ individuo-1) de las plantas evaluadas en cada población son consecuencia de las condiciones climáticas y topográficas diversas de las zonas áridas, que generan patrones espaciales y temporales en la distribución de las especies, como es el caso de candelilla (Tapia-Tapia y Reyes-Chilpa, 2008; Arato, Speelman y Van, 2014). Estos patrones se pueden también observar en otras especies como Prosopis laevigata (Humb. \& Bonpl. Ex Willd.) M.C. Johnst., que presentaron variaciones de la biomasa aérea de individuos con similares dimensiones y de diferente procedencia en el Noreste de México, como resultado de las condiciones ambientales del sitio y a la aplicación de actividades silvícolas en un pasado (Méndez et al., 2012). De igual manera en plantaciones de Eucalyptus grandis W. Hill ex Maiden en el noreste de Argentina, se determinó que la zona agroecológica influye en la acumulación de biomasa aérea y en la distribución de ésta en sus componentes (Winck et al., 2015).

Navar-Chaídez (2010) y Yepes et al. (2015) mencionan que los mejores estadísticos de ajuste de ecuaciones alométricas para estimar biomasa se obtienen al ajustarlas a escala de sitio o por especie, lo que reduce el número de observaciones y consigo la varianza de la muestra. Esto llevó a clasificar las poblaciones de muestreo en tres grupos diferentes para así obtener un mejor ajuste de los modelos y aplicación de los resultados al estimar la Btv. Para la clasificación de la muestra se debe realizar la selección correcta de las variables independientes para el ajuste de los modelos, con ello se mejoran los estadísticos y se reducen los residuales en las estimaciones; además, estas variables deben de ser de fácil medición (Rodríguez-Ortiz et al. 2012; Ferrere et al. 2014; Winck et al., 2015).

Los mejores modelos para estimar la Btv incluyeron las variables DPB, DPC y A de la planta, con valores de coeficiente de determinación superiores a 87\%. Resultados similares han sido publicados en otros trabajos como el de Solano et al. (2014), quienes obtuvieron una explicación de la varianza de los datos superior a $70 \%$ al incluir las variables diámetro normal y altura comercial en 19 modelos para estimar la biomasa aérea de 19 especies arbóreas del bosque seco pluviestacional. Por su parte, VillavicencioGutiérrez et al. (2018) indicaron que las variables como el diámetro promedio de la cobertura, la altura total y la interacción entre ambos presentaban una relación significativa con la biomasa foliar seca de Lippia graveolens Kunth, con valores de coeficiente de determinación de $82 \%, 53 \%$ y 83\%, respectivamente. Para Prosopis laevigata, Méndez et al. (2012) indicaron que el diámetro basal está altamente correlacionado con la biomasa aérea de la planta y el ajuste estadístico de modelos mejoró al ingresar una segunda variable dasométrica, la altura de la planta (Flores et al., 2018).

Se encontró que los modelos seleccionados por región para estimar la Btv explican el 92\%, 86\% y 64\% de la variabilidad de los datos de campo para GI, GII y GIII, respectivamente, lo que indica un ajuste adecuado. Resultados similares para especies forestales no maderables se han obtenido por Valencia, Sánchez, Quiñones y Martínez (2003), quienes obtuvieron, para cuatro especies del norte de México, valores del coeficiente de determinación ajustado de 89\% para Agave lechuguilla, 62\% para L. graveolens Kunth., 83\% para P. laevigata y 63\% para E. antisyphilitica, este valor bajo para candelilla es similar al aquí obtenido para la región de Sierra Mojada (GIII). Por su parte Méndez et al. (2012), en modelos ajustados por sitio para estimar la biomasa de $P$. laevigata, obtuvieron coeficientes de determinación superiores a 91\%. Villavicencio-Gutiérrez et al. (2018) registraron valores de $81 \%$ con el modelo de Schumacher-Hall, para estimar la biomasa foliar seca de L. graveolens Kunth., en poblaciones naturales distribuidas en el sureste de Coahuila.

\section{CONCLUSIONES}

La procedencia de la muestra, ya sea por población o por municipio, presentó diferencias significativas en cuanto a la biomasa por individuo.

El modelo de Schumacher-Hall presentó mejores ajustes para estimar la biomasa verde en las regiones comprendidas en los grupos GI (modelo 2) y GII (modelos 1 y 2), utilizando como variables independientes el diámetro 
promedio de la cobertura, diámetro promedio de la base y la altura promedio de la planta. Para el tercer grupo (GIII) el modelo de mejor ajuste fue el de Schumacher de tipo exponencial (modelo 7), empleando el diámetro promedio de la cobertura como variable independiente, sin embargo, se requiere mayor muestra para mejorar el ajuste en el modelo propuesto.

Estas herramientas serán útiles para evaluar con mayor precisión la biomasa verde de candelilla, que es utilizada en los estudios técnicos justificativos para el aprovechamiento forestal, asegurando un menor sesgo en las estimaciones totales.

\section{REFERENCIAS}

Arato, M., Speelman, S., \& Van, H. G. (2014). The contribution of non-timber forest products towards sustainable rural development: The case of Candelilla wax from the Chihuahuan Desert in Mexico. Natural Resources Forum, 38(2), 141-153. doi: 10.1111/1477-8947.12043

Aristizábal, D. J. (2011). Desarrollo de modelos de biomasa aérea en combríos de cafeto (Coffea arabica L.) mediante datos simulados. Rev. U.D.C.A Actualidad \& Divulgación Científica, 14(1), 49-56.

Carrillo, F., Acosta, M., Flores, E., Juárez, J. E., \& Bonilla, E. (2014). Estimación de biomasa y carbono en dos especies arbóreas en La Sierra Nevada, México. Revista Mexicana de Ciencias Agrícolas, 5(5), 779-793. doi: 10.29312/remexca.v5i5.901

Cervantes, M. C. (2002). Plantas de importancia económica en las zonas áridas y semiáridas de México. Temas Selectos de Geografía de México (pp. 125-137). México, D.F.: Universidad Nacional Autónoma de México. .

Challenger, A. \& Soberón, J. (2008). Los ecosistemas terrestres, en Capital natural de México, vol. I: Conocimiento actual de la biodiversidad (pp. 87-108). México: Comisión Nacional de Biodiversidad (Conabio).

Chen, J. J. (1978). Testing for outliers in linear models. Retrospective these and disseartations. Recuperado de http://lib.dr.iastate.edu/rtd/6448

Coenders, G. \& Saez, M. (2000). Collinearity, heteroscedasticity and outlier diagnostics in regression. Do they always offer what they claim? En A. Ferligoj \& A. Mrvar (Eds.), New approaches in applied statistics, Metodoloski Zvezki 16 (pp.79-94). Ljubljana: FDV
Ferrere, P., Lupi, A. M., \& Boca, R. T. (2014). Estimación de la biomasa aérea en árboles y rodales de Eucalyptus viminalis Labill. Rev. Ciencias Forestales-Quebracho, 22(1), 100-113.

Flores, M. F., Vega-Nieva, D. J., Corral-Rivas, J. J., ÁlvarezGonzález, J. G., Ruiz-González, A. D., López-Sánchez, C. A., \& Carrillo, A. (2018). Desarrollo de ecuaciones alométricas de biomasa para la regeneración de cuatro especies en Durango, México. Revista Mexicana de Ciencias Forestales, 9(46), 157-185. doi:10.29298/rmcf.v9i46.119

Gómez-García, E., Crecente-Campo, F., \& Diéguez-Aranda, U. (2013). Tarifas de biomasa aérea para abedul (Betula pubescens Ehrh.) y roble (Quercus robus L.) en el noreste de España. Madera y Bosques, 19(1), 71-91. doi: 10.21829/myb.2013.191348

Gyenge, J., Fernández, M. E., Sarasola, M., Urquiza, M., \& Schlichter, T. (2009). Ecuaciones para la estimación de biomasa aérea y volumen de fuste de algunas especies leñosas nativas en el valle del río Foyel, NO de la Patagonia Argentina. Bosque, 30(2), 95-101. doi: 10.4067/S071792002009000200005

Hernández-Ramos, J., Santos-Posadas, H. M., Valdez-Lazalde, J. R., Tamarit-Urias, J. C., Ángeles-Pérez, G., HernándezRamos, A., Peduzzi, A., \& Carrero, O. (2017). Biomasa aérea y factor de expansión en plantaciones forestales comerciales de Eucalyptus urophylla S. T. Blake. Agrociencia, 51(8), 921-938.

Instituto Nacional de Estadística, Geografía e Informática [Inegi]. (2007). Conjunto de datos vectoriales escala 1:1000000, Unidades climáticas. Recuperado de http:/ /www.beta.inegi.org.mx/app/biblioteca/ficha.html? upc $=702825267551$

Instituto Nacional de Estadística, Geografía e Informática [Inegi]. (2016). Conjunto de datos vectoriales de Uso del suelo y vegetación. Escala 1:250 000. Serie VI. Capa Unión. Recuperado de http:/ /www.beta.inegi.org.mx/app/biblioteca/ficha.html? upc $=889463173359$

Lambert, M. C., Ung, C. H., \& Raulier, F. (2005). Canadian national tree aboveground biomass equiations. Canadian Journal of Forest Research, 35(8), 1996-2018. doi: 10.1139/X05112

Limones-Rubio, D., Aguirre-Ureña, E. A., Fonseca-Espino, J., Muro G., \& Sánchez, J. (2015). Aprovechamiento de los recursos maderables y no maderables de la zona semiárida de Durango. Revista Ciencia UANL, 18(74), 73-77.

Méndez, G. J., Turlan, O. A., Ríos, J. C., \& Nájera, J. A. (2012). Ecuaciones alométricas para estimar biomasa aérea de Prosopis laevigata (Humb. \& Bonpl. Ex Willd.) M.C. Johnst. Revista Mexicana de Ciencias Forestales, 3(13), 57-72. 
Návar-Cháidez, J. J. (2010). Biomass allometry for tree species of northwestern Mexico. Tropical and Subtropical Agroecosystems, 12(3), 507-519.

Organización de las Naciones Unidas para la Agricultura y la Alimentación [FAO] (2004). Estudio de tendencias y perspectivas del Sector Forestal en América Latina Documento de Trabajo: Informe Nacional México.

Rodríguez-Ortiz, G., Santos-Posadas, H. M., GonzálezHernández, V. A., Aldrete, A., Gómez-Guerrero, A., \& Fierros-González, A. M. (2012). Modelos de biomasa aérea y foliar en una plantación de pino de rápido crecimiento en Oaxaca. Madera y Bosques, 18(1), 25-41. doi: 10.21829/myb.2012.1811116

Secretaria de Medio Ambiente y Recursos Naturales [Semarnat]. (2016). Anuario estadístico de la producción forestal 2016. Semarnat. Recuperado de https://www.gob.mx/cms/uploads/attachment/file/2829 51/2016.pdf

Secretaria de Medio Ambiente y Recursos Naturales [Semarnat]. (2013). Anuario estadístico de la producción forestal 2013. Semarnat. Recuperado de https://www.gob.mx/cms/uploads/attachment/file/2829 27/2013.pdf

Solano, D., Vega, C., Eras, V. H., \& Cueva, K. (2014). Generación de modelos alométricos para determinar biomasa aérea a nivel de especies, mediante el método destructivo de baja intensidad para el estrato de bosque seco pluviestacional del Ecuador. CED AMAZ, 4(1), 32-44.

Soriano-Luna, M. A., Ángeles-Pérez, G., Martínez-Trinidad, T., Plascencia-Escalante, F. O., \& Razo-Zárate, R. (2015). Estimación de la biomasa aérea por componente estructural en Zacualtipán, Hidalgo, México. Agrociencia, 49(4), 423-438.

Statistical Analysis Systems [SAS] Institute Inc. (2017). SAS/STAT(R) 9.22 User's Guide. Recuperado de https://support.sas.com/documentation/cdl/en/statug/6 3347/HTML/default/viewer.htm\#statug_anova_sect001. htm

Tapia-Tapia, E. C. \& Reyes-Chilpa, R. (2008). Productos forestales no maderables: aspectos económicos para el desarrollo sustentable. Madera y Bosques, 14(3), 95-112. doi: 10.21829/myb.2008.1431208

Tlaxcala-Méndez, R. M., Santos-Posadas, H. M., Hernández-de la Rosa, P., \& López-Ayala, J. L. (2016). Variación del factor de forma y el ahusamiento en procedencias de cedro rojo (Cedela odorata L.). Agrociencia, 50(1), 89-105.

Trincado, G. \& Leal, C. (2006). Ecuaciones locales y generalizadas de altura-diámetro para pino radiata (Pinus radiata). Bosque, 27(1), 23-34. doi: 10.4067/S071792002006000100003

Valencia, C. C., Sánchez, M. H., Quiñones, V. J., \& Martínez, R. J. (2003). Predicción de la biomasa aérea de cuatro especies arbustivas del semidesierto en la Comarca Lagunera. Agrofaz, 3(2), 365-372.

Villavicencio-Gutiérrez, E. E., Hernández-Ramos, A., AguilarGonzález, C., \& García-Cuevas, X. (2018). Estimación de la biomasa foliar seca de Lippia graveolens Kunth del sureste de Coahuila. Revista Mexicana de Ciencias Forestales, 9(45), 187207. doi: 10.29298/rmcf.v9i45.139

Volke, H. V. (2008). Estimación de funciones de respuesta para información de tipo no experimental, mediante regresión (1a ed.). Montecillo, Estado de México, México: Colegio de Postgraduados.

Williams, L. J. \& Abdi, H. (2010). Fisher's Least Significant Difference (LSD). Encyclopedia of Reserch Design. Oak, EU: SAGE.

Winck, R., Fassola, H., Barth, S., Crechi, E., Keller, A., Videla, D., \& Zaderenko, C. (2015). Modelos predictivos de biomasa aérea de Eucalyptus grandis para el noreste de Argentina. Ciencia Forestal, 25(3), 595-606. Doi: $10.5902 / 1980509819611$

Yepes, A., Zapata, M., Bolivar, J., Monsalve, A., Milena, S., SierraCorrea, P., \& Sierra, A. (2016). Ecuaciones alométricas de biomasa aérea para la estimación de los contenidos de carbono en manglares del Caribe Colombiano. Biología Tropical, 64(2), 913-926.

Zamora, M. C. M., Velasco, B. E., Cano, P. A., \& Arellano, R. A. (2008). Manual que establece de los criterios técnicos para el aprovechamiento sustentable de los recursos forestales no maderables de clima árido y semiárido. Semarnat-Inifap.

Manuscrito recibido el 19 de junio de 2018

Aceptado el 15 de enero de 2019

Publicado el 16 de octubre de 2019

Este documento se debe citar como:

Hernández-Ramos, A., Cano-Pineda, A., Flores-López, C., HernándezRamos, J., García-Cuevas, X., Martínez-Salvador, M., \& Martínez Á., L. (2019). Modelos para estimar biomasa de Euphorbia antisyphilitica Zucc. en seis municipios de Coahuila. Madera y Bosques, 25(2), e2521806. doi: 10.21829/myb.2019.2521806

Madera y Bosques por Instituto de Ecología, A.C. se distribuye bajo una Licencia Creative Commons Atribución-NoComercialCompartirlgual 4.0 Internacional. 